\title{
Cultura organizacional: algunas reflexiones a la luz de los nuevos retos
}

\author{
Pérez Martínez, Armenio*
}

\section{Resumen}

El artículo "La cultura organizacional, algunas reflexiones a la luz de los nuevos retos", tiene como objetivo analizar el concepto de cultura organizacional a partir de la situación contemporánea de las organizaciones, así como describir la relación entre la cultura organizacional y la estrategia organizacional. Para ello se utilizan los métodos teóricos histórico-lógico para el estudio de los antecedentes conceptuales, análisis-síntesis y dialéctico a la hora de realizar los análisis. Como resultados fundamentales resaltan algunas características comunes e invariables dentro del concepto de la cultura organizacional, lo cual permite una valoración desde el punto de vista epistemológico en la posibilidad de concebir nuevos puntos de enfoque del tema de la cultura en las organizaciones, así como en la relación cultura y estrategia organizacional.

Palabras clave: Cultura organizacional, estrategia organizacional, roles organizacionales.

\section{Organizational Culture: Some Reflections in the Light of New Challenges}

\section{Abstract}

The objective of the article "Organizational Culture: Some Reflections in the Light of New Challenges" is to analyze the concept of organizational culture, based on the contemporary situation of organizations, as well as to describe the relationship between organizational culture and organizational strategy. To accomplish this, historical-logical theoretical methods were used to study the conceptual antecedents; analysis-synthesis and dialectical methods were used when performing the analyses. Fundamental results show that some common and invariable characteristics in the concept of organizational culture stand out permitting a valuation from the epistemological viewpoint of the possibility of conceiving new focal points for the topic of culture in organizations, as well as the relationship between organizational culture and organizational strategy.

Key words: Organizational culture, organizational strategy, organizational role.

\section{Recibido: 15-05-08. Aceptado: 10-02-09}

Master en Gestión Turística y Licenciado en Psicología. Centro de Estudio de Dirección, Universidad de Ciego de Ávila.E-mail:armenito@economia.unica.cu,perez_armenio@yahoo.es 


\section{Introducción}

El mundo contemporáneo puede ser definido como un escenario gigantesco con actores y actrices interdependientes. A partir de 1985, comienza el nacimiento de una nueva era, definida desde que Bill Gates lanzó Windows, que ayudó a eliminar las fronteras, hacer más rápidas las comunicaciones, aparejado a otros avances tecnológicos y comunicativos. También, la caída del campo socialista europeo es otro de los acontecimientos presentes a fines de la década de 1980. Además la reunión del Hotel Plaza (Nueva York, USA), donde se celebró la Cumbre del G-5 y se tomaron importantes acuerdos sobre el comercio internacional, marcó un viraje en las relaciones entre los países a nivel global; así como la apertura de China al comercio internacional, hacen de estas últimas décadas marquen un viraje en las relaciones entre los Estados, las sociedades, las organizaciones y el propio individuo (Ohmae, 2005). Al señalar estos aspectos se debe hacer referencia al desarrollo científico y tecnológico (Nuñez, 2003) que se ha venido gestando desde fines de la década de 1960 y que dan lugar al conocido enfoque de ciencia, tecnología y sociedad, de donde se han derivado todos los avances ulteriores. En opinión Castro (2007:3) "El mundo de hoy es muy diferente al mundo que Marx y Lenin conocieron; nadie pudo conocerlo, nadie pudo imaginar las comunicaciones en cuestión de segundos. Vieron la globalización, vieron a lo que conducía un sistema donde las fuerzas productivas se desarrollaban, vieron que el desarrollo de las fuerzas productivas alcanzaría tales niveles que produciría en el mundo situaciones nuevas, grandes cambios".

En este contexto es que las organizaciones sociales, lucrativas o no, enfrentan nuevos retos, como nunca antes, en su devenir histórico. En la actualidad las organizaciones se debaten para su propia subsistencia en una encarnizada e insostenible carrera competitiva, que trasciende la ya conocida lucha por los segmentos de mercado cada vez más fraccionados y diferenciados entre sí, sino que la disputa es por la credibilidad social, perfeccionar la gestión de su capital intelectual, elevar la calidad de vida de la comunidad medioambiental y cultural, así como por trascender la inmediatez de los planes para estimular las proyecciones de las estrategias organizacionales.

Aún así, se encuentra en la bibliografía de la ciencia administrativa contemporánea, algunas falacias que hacen afirmar la existencia ilimitada de las organizaciones sociales (Schein, 1986; Chiavenato, 1995). Este aspecto, comprendido fuera de un certero análisis epistemológico, apegado a la realidad, en la crítica y la ética; parecería veraz ya que la aparición de las micro, pequeña y mediana empresas florecen en la actualidad; pero nacen con una esperanza de vida corta, ya que en la competencia pierden sus principales armas: su capital humano y lo que este aporta a la organización; tampoco trascienden las formas organizativas de las empresas de mayor desarrollo tecnológico y social, es decir, su gestión se mantiene siendo copia fiel de las "empresas de éxito". Este hecho afecta a todas las organizaciones que en estos momentos no generen formas de perfeccionar su 
gestión y elevar el compromiso de sus recursos humanos.

Específicamente la cultura organizacional es el objeto de análisis en esta ocasión. La realidad impone esta categoría científica como un arma estratégica de las organizaciones contemporáneas. Su análisis y conocimiento permite mejorar aspectos medulares en la vida de la institución, tanto desde el punto de vista humano como tecnoproductivo. Ante las incertidumbres y oportunidades vigentes, emerge como catalizador de soluciones y decisiones trascendentales, ya que es un elemento característico y determinante en la organización.

Este artículo persigue trasmitir una valoración del concepto de cultura organizacional a partir de sus principales representantes; llegando a interrogantes que hoy son inevitables cuando de este tema se está debatiendo. Otros aspectos serán examinados y que resultarán de interés. Algunos aportes teórico-práctico resultan de estas valoraciones y pueden dar lugar a nuevos, profundos e interesantes debates al respecto.

\section{Antecedentes históricos del estudio de la cultura organizacional}

Los antecedentes del estudio de la cultura organizacional pueden ser ubicados en los orígenes de las ciencias sociales. Entender esta contribución es muy importante porque muchos de los dilemas y debates que se dan en torno de la cultura organizacional reflejan las diferencias en la tradición histórica de la investigación social.
Dentro de las ciencias sociales se aprecia que los aportes de mayor trascendencia son los de la antropología, la sociología, la psicología social y la economía. Todas desde diferentes aristas investigan el comportamiento del ser humano dentro de distintos grupos sociales y con diferentes funciones, donde la cultura ha estado presente como un resultado de las relaciones interpersonales.

La influencia de la Economía ha sido menor que la de las disciplinas anteriormente descritas, sin embargo los análisis económicos ven en la cultura organizacional una herramienta que puede ser usada para incrementar ganancias. Ouchi (1982) y Peters y Waterman (1984) buscan en las explicaciones culturales el éxito financiero. Ellos definen excelencia en parte, como un resultado financiero consistente y de alto rendimiento. Con la aparición de la ciencia administrativa a inicios del siglo XX, se abre un nuevo espacio para el estudio de la cultura organizacional, más profundo desde el punto de vista epistemológico, a la vez, más holístico y sistémico, llevando no solo a propuestas teóricas, sino que ha trascendido el discurso científico y se ha nutrido de la acción, potenciando la relación con otras ciencias como las señaladas con anterioridad.

El concepto de cultura aplicado a la organización se fue gestando desde el aporte de la escuela de administración de las relaciones humanas, a partir de los experimentos desarrollados por Elton Mayo, se empiezan a reconocer los aspectos subjetivos e informales de la realidad organizacional. Mayo (1972), se interesó por indagar acerca de los factores 
que inciden en el desempeño del trabajador, llegando a la conclusión que el ambiente del grupo al cual pertenece el individuo incide significativamente en la percepción que éste tiene acerca de los aspectos objetivos de la organización.

\section{Algunos conceptos de cultura organizacional}

Teniendo en cuenta su naturaleza y complejidad, no existe una definición totalmente aceptada. Nuestra opinión hace coincidir este análisis con otros similares de conceptos que han partido de ciencias primarias, que al compartir parte de su objeto de estudio con otras ciencias; además de apoyarse en trabajos de investigadores de otra rama del conocimiento, no han logrado integrar un concepto coherente para la ciencia que en la actualidad responde a la categoría que se precisa (Bunge, 2004).

Para una mejor comprensión del concepto que se está analizando se realizará la muestra, en primer lugar del concepto de cultura para posteriormente mostrar algunos conceptos más difundidos de cultura organizacional, los que si bien no son totalmente contrapuestos, si marcan puntos críticos nuevos que este autor ha decidido subrayar. (Los destacados son de este autor, con el objetivo de llamar la atención sobre aspectos medulares del concepto de cultura organizacional).

La Real Academia Española (2001: 309) define cultura, como término general, no específicamente organizacional, como "resultado o efecto de cultivar los conocimientos humanos y de afinarse pormedio del ejercicio las facultades inte- lectuales del hombre" y como "conjunto de modos de vida y costumbres, conocimiento y grado de desarrollo artístico, científico, industrial, en una época o grupo social".

Para Geertz (1987:103) "la cultura es un patrón de significados transmitidos históricamente, incorporados en símbolos, un sistemas de concepciones heredadas y expresadas en forma simbólica por medio de las cuales los hombres comunican, perpetúan y desarrollan su conocimiento y sus actividades con relación a la vida". Este criterio vuelve a hacer referencia a la cultura de manera general, como en el caso anterior, pero no deja de brindar elementos que resultan necesarios tomar en cuenta.

Peters y Waterman (1984) dicen que la cultura organizacional es un "conjunto dominante y coherente de valores compartidos transmitidos por significados simbólicos como cuentos, mitos, leyendas, slogans y anécdotas". Por su parte, Deal y Kennedy (1982) la definen la cultura organizacional como "la forma en que nosotros hacemos las cosas aquí".

Fleury, en el libro Cultura y poder en las Organizaciones, señalaba a la necesidad de añadir la arista política en el concepto de cultura, por considerar que el poder es uno de sus aspectos inherentes. Para la autora la cultura es "un conjunto de valores y supuestos básicos expresados en elementos simbólicos, que en su capacidad de ordenar, atribuir significados, construir la identidad organizacional, tanto actúan como elemento de comunicación y consenso, como ocultan e instrumentalizan las relaciones de dominio" (Fleury, 1989:22). 
Aguirre (1996:42) define la cultura organizacional como: "Conjunto de elementos interactivos fundamentales, compartidos grupalmente, sedimentados a lo lago de la vida de la empresa a la cual identifican, por lo que son trasmitidos a los nuevos miembros, y que son eficaces para la resolución de problemas". Chiavenato (1995:464), destacado investigador y autor de importantes obras de administración presenta la cultura organizacional como "...un modo de vida, un sistema de creencias y valores, una forma aceptada de interacción y relaciones típicas de determinada organización".

García y Dolan (1997:33) se destacan como iniciadores de la Dirección por Valores, temática que necesita comprenderse en el marco de la cultura organizacional, que estos autores definen como “... la forma característica de pensar y hacer las cosas... en una empresa... por analogía es equivalente al concepto de personalidad a escala individual...".

En Cuba, se aprecia una definición clara en la labor. Díaz (2006:21) plantea que: "Cultura son las creencias, ya sean inventadas, desarrolladas o descubiertas, que los grupos juzgan como válidas y le permiten adaptarse al entorno e integrarse internamente, así como que son enseñados como el modo correcto de percibir, pensar y sentir sus problemas".

La definición más aceptada de Cultura Organizacional por los investigadores es la de Schein (1986:56), al referir que: "Cultura organizacional es el patrón de premisas básicas que un determinado grupo inventó, descubrió o desarrolló en el proceso de aprender a resolver sus problemas de adaptación externa y de integración interna y que funcionaron sufi- cientemente bien a punto de ser consideradas validas y, por ende, de ser enseñadas a nuevos miembros del grupo como la manera correcta de percibir, pensar y sentir en relación a estos problemas.

A pesar de esta real diversidad hay algunos atributos comunes que emergen. Primero, todas las definiciones se refieren a algún conjunto de valores mantenido por individuos en una organización. Esos valores definen cuál es el comportamiento bueno o aceptable y ayudan a los integrantes de una organización a entenderla y a saber cómo actuar en ella. Segundo, los valores que conforman la cultura organizacional más que estar escritos o definidos están implícitos en la organización y deben articularse con los valores y creencias personales de los empleados. Tercero, las definiciones tienen un marcado énfasis en los significados simbólicos a través de los cuales los valores son comunicados.

Parece que existe un amplio acuerdo en que la cultura organizacional se refiere a un sistema de significado compartido entre sus miembros, y que distingue a una organización de las otras (Hatch, 1993).

Díez (1999, 39-40) recoge los elementos comunes en la mayoría de las corrientes y definiciones y termina con la definición-síntesis de quinientas definiciones de cultura organizacional:

1. La cultura es un fenómeno social.

2. El comportamiento cultural obedece a pautas.

3. La cultura proporciona un modelo de vida. Cultura ideal, cultura modal.

4. La cultura no es fruto de la herencia genética, sino herencia tradicional o social históricamente acumulada. Re- 
sulta de dos procesos de aprendizaje: socialización (integración de un individuo en un grupo), e inculturación (interiorización de los modelos de conducta propuestos por el grupo).

5. No se puede identificar la cultura con la conducta. Ésta es consecuencia de la cultura. También hay otros factores que influyen en la conducta.

6. No se puede identificar la cultura con la tradición.

7. Toda cultura es un sistema de expectativas.

8. La cultura no es un todo coherente y armónico. Existen contraindicaciones internas, está abierta al cambio.

Smircich (1983), identifica las metáforas predominantes que han surgido en los intentos por comprender la cultura organizacional: cultura como una variable externa, como una variable interna y cultura como una metáfora raíz. En el primer caso se hace hincapié en la influencia que ejerce la sociedad en la cultura organizacional. En este sentido las organizaciones son manifestaciones de sistemas culturales más amplios. Los miembros traen a la organización otras pautas culturales que se van adaptando o no al funcionamiento de la empresa. El segundo caso se entiende a las organizaciones como fenómenos que producen cultura; son vistas como instrumentos sociales que producen bienes, servicios y subproductos tales como artefactos culturales. La propia cotidianidad va formando la cultura a través de los integrantes de las organizaciones. En el tercer caso se entiende que la cultura no es algo que las organizaciones tienen, sino es algo que las organizaciones son, "Las organizaciones son entendidas y analizadas no principalmente en términos económicos o materiales, sino en términos de sus aspectos expresivos y simbólicos" (Smircich, 1983:347).

En nuestro criterio, asumir cualquiera de estas tres posiciones sólo haría menoscabar el alcance del concepto de cultura organizacional. Esta se encuentra relacionada estrechamente con otras organizaciones dentro de la estructura social a la que pertenecen y además de intercambiar bienes también comparten patrones culturales. Los sujetos que componen la organización son los que se encargan de validar en su actuación diaria los elementos que integral la cultura, así como la van construyendo. Al considerar que las organizaciones en sí mismas son cultura, estamos logrando rescatar y poner en su justo lugar el papel de las interacciones humanas y la historia de la organización.

A partir del análisis realizado, se considera que se pudieran establecer algunos aspectos básicos dentro del concepto de cultura organizacional. Los mismos pueden proporcionar una mejor comprensión teórico-metodológica, ya que sirven tanto a investigadores como a directivos para la realización de sus funciones:

- La cultura no se puede manejar por parte de los directivos para conseguir resultados, metas, fines u objetivos. Es un sistema relativamente estable de significados y sentidos compartidos, difíciles de manipular, totalmente ajena a una perspectiva instrumental.

- Interpretar la cultura implica subjetividad, que debe ser consciente y expli- 
citada. No se puede interpretar algo sin una concepción teórica previa que nos permita analizar y explicar la realidad.

- La cultura organizacional, a pesar de ser relativamente estable, no es estática, sino dinámica: está construyéndose continuamente con sus propios mecanismos, incluso sin que haya habido modificaciones en sus componentes. El cambio y las nuevas experiencias nutren a la cultura organizacional de nuevos elementos que se configuran con los contenidos subjetivos ya existentes.

- La cultura organizacional, como sistema trasciende la sumatoria de elementos aislados que la componen, no es una suma de variables o de partes que se puedan analizar por separado, pues el análisis sería sesgado, parcial al eliminar aspectos que no se pueden olvidar aislar. Sistema "como definición conceptual para describir una pauta de relaciones, más que para referirnos a una suma de partes percibidas o encontradas" (Katz y Kahn, 1986:28). Este aspecto es de vital importancia a la hora de estudiar alguno de sus elementos componentes (valores, actitudes, creencias, normas, comportamiento, entre otros), ya que no puede realizarse ajeno a la cultura de la que forma parte.

- En una organización no se da una cultura organizacional única, ni están unidos sus integrantes que todos piensen lo mismo y estén de acuerdo en todo. Siempre hay pequeños grupos (subculturas) con interrelaciones e interacciones constantes.
- Para investigar la cultura hay que realizar una inmersión en la organización, ser uno más dentro de ella. Sólo así se puede comprender la realidad y sólo así se puede realizar el cambio desarrollador, que no es posterior a la investigación, sino conjunto, coetáneo, de tal manera que llega a ser difícil cuándo se está investigando y cuándo se está produciendo el cambio. "La teoría de los sistemas dicta una estrategia para la investigación, que se encuentra en oposición fundamental al reduccionismo o paso inmediato hacia algún nivel más elemental para que sea posible comprender los fenómenos sociopsicológicos. El primer paso será siempre ir al siguiente nivel superior de la organización del sistema, para estudiar la dependencia del sistema en cuestión respecto al suprasistema del que es parte, pues este asienta los límites de varianza de conducta del sistema dependiente" (Katz y Kahn, 1986:54).

- La cultura es un sistema abierto, recibe influencia de otros sistemas culturales. Erróneamente se considera que el sujeto que se incorpora de manera pasiva a la organización asume la cultura existente como propia, sin embargo, todas las personas participan en otras organizaciones e incluso en la sociedad, donde se puede decir que existe un intercambio de contenidos culturales. Este aspecto se ejemplifica en empresas nuevas, donde se aprecian rasgos culturales de organizaciones de otro tipo, siendo estas últimas coincidentes con contenidos de las culturas de las or- 
ganizaciones de las que proceden estos recursos humanos.

- La cultura organizacional, se manifiesta a través de un proceso de doble formación. Los contenidos antes de consolidarse como parte de la cultura y pasar por un proceso de interiorización, tienen un momento de vida social, real y actual, en la acción cotidiana de la vida organizacional donde se pueden reestructurar algunos contenidos ya existentes, así como complementar o validar otros. Hoy, en el funcionamiento organizacional, se consolida la cultura existente, se enriquece, a la vez que es interiorizada. Este elemento será retomado posteriormente para resaltar la necesidad de relacionar la cultura organizacional con la estrategia de desarrollo de la organización.

La cultura desempeña diversas funciones dentro de una organización. En primer lugar, acumula los contenidos y experiencias de una organización a través de su historia; en segundo lugar tiene un papel de definición de límites; es decir, crea diferencias entre una organización y las demás. En tercer lugar, conlleva un sentido de identidad para los miembros de la organización. En cuarto lugar, la cultura facilita la generación del compromiso con algo más grande que el interés personal del individuo. En quinto lugar, mejora la estabilidad del sistema social. La cultura es el pegamento social que ayuda a mantener unida a la organización, al proporcionar normas apropiadas para lo que deben hacer y decir los empleados. En sexto lugar la cultura tiene que guiar las proyecciones y estrategias de la organi- zación, siendo un elemento a partir del cual se logren los resultados esperados. Por último, la cultura sirve como mecanismo de control y de sensatez que guía y modela las actitudes y el comportamiento de los empleados.

La cultura también ha sido percibida como un elemento que limita las organizaciones, describiendo la existencia de tres barreras fundamentales: barrera al cambio, a la diversidad y a las fusiones y adquisiciones (Smircich, 1983). Se considera por nuestra parte que el papel de la cultura nunca estará en detrimento del desarrollo organizacional, se plantea que pueden existir culturas rígidas que sí frenan, pero esta es tomada en cuenta en la Dirección Estratégica de la organización, si se construye y se tiene en cuenta diariamente, difícilmente será un elemento que aplaque el cambio, sino será abierto a este. Si la cultura organizacional es vista como pasado, como un resultado y no como un proceso, puede considerarse como limitante de aspectos actuales y vitales de la empresa, ya que es una estructura estática. Ahora bien, teniendo en cuenta el proceso de doble formación, su vigencia y potencialidades, la cultura puede facilitar todos los procesos y metas que se propongan en la organización, ya que ella está presente siempre en la cotidianidad de la institución social.

El análisis anterior por los derroteros de la cultura organizacional ha permitido que se propongan al respecto nuevas ideas. Partiendo de la aceptación de las generalidades de los autores anteriores y concientes de lo inacabado de este concepto, se percibe la existencia de un nuevo elemento que no ha sido tomado en 
cuenta a la hora de valorar la cultura organizacional, así como el papel del sujeto concreto dentro de la cultura, ya que a través de este es que la primera se manifiesta y para su estudio es necesario partir de las construcciones compartidas por los individuos de su realidad organizacional.

La manifestación de la cultura en las organizaciones pasa por el prisma del rol que juega el individuo dentro de la institución social. La forma en que el individuo interactúa con la cultura y con los demás miembros de la organización es a través del papel que este desempeña en la empresa a la hora de realizar su función social. Los roles "describen las formas específicas de conducta asociada con determinadas tareas; en un principio estas surgen de los requerimientos de la tarea... tales papeles son pautas estandarizadas de conducta que se piden a toda persona que desempeñe una tarea en relación funcional dada, sin tener en cuenta sus deseos particulares u obligaciones" Katz y Kahn, 1986:44).

Esta realidad, según nuestra opinión, tiene una doble implicación para la investigación científica. En los modelos que pretenden explicar la cultura organizacional desde el sujeto (Mayo, 1972; Díaz, 2006), única forma en que esta puede ser estudiada, pues de manera ajena a estos no se manifiesta como un elemento sistémico y con vida propia, este elemento movilizador del comportamiento y de las interpretaciones de los miembros de la organización tiene que estar presente, ya que determina la función individual dentro de la organización. Esta interrelación entre cultura y roles permite salvar una vieja deuda teórica con la categoría organización (Schein, 1986; Deal y Ken- nedy, 1982). Esta última, si se analiza desde un enfoque de sistema, deja de ser una suma aisladas de esfuerzos, voluntades y tareas (roles), para apoyarse en la cultura y formar un esquema monolítico de roles definidos en función de objetivos y con una vida e historia propia.

Siguiendo un análisis funcionalista, desde el punto de vista práctico este aspecto al que se ha hecho referencia con anterioridad, permite al investigador partir de las funciones de cada sujeto, que además de portar la cultura organizacional, la pone en función de su tarea y en esta misma práctica es donde se realizó el proceso de interiorización con la cultura. No es igual la apropiación y manifestación de la cultura organizacional en un sujeto que su función la desarrolle ajeno al contacto con el personal a uno que se encuentre en contacto constante y directo. También se aprecian diferencias sustanciales entre los que realizan funciones de producción y los de apoyo, etc.

Incluir el rol en el análisis de la cultura organizacional conlleva a desarrollar un análisis acertado, aunque con anterioridad se ha venido desarrollando de alguna manera en diversos estudios (Schein, 1986). La cultura organizacional no es "algo" que compartimos o no cuando se forma parte de una organización, ya que esta se pone de manifiesto según el rol que usted desempeñe en la organización, al permitirle interiorizar y construir la cultura y a la vez, ponerla de manifiesto en sus comportamientos.

\section{Estrategia y cultura}

Al incluir el rol y su influencia dentro de los aspectos necesarios a analizar en la 
cultura organizacional, se considera que se ha dado otro paso en el acercamiento de los elementos formales de la organización con los elementos subjetivos. Este hecho permite comprender aun mejor la posible relación entre estrategia y cultura organizacional, elemento que en nuestra opinión no ha sido estudiado en su total magnitud, criterio reconocido por Díaz (2006); en el cual estriba gran parte del éxito organizacional, no solo desde el punto de vista financiero, sino desde las aristas de la sostenibilidad y la competitividad.

Muchas compañias han descubierto que pueden concebir estrategias nuevas válidas desde el punto de vista financiero, productivo o mercantil, pero que en cambio no pueden implantarlas porque las presunciones, valores y métodos de trabajo que requieren para ello no guardan ninguna correspondencia con las presunciones fundamentales de las empresas.

El término cultura se usa hoy exactamente y se ofrece como explicación válida de diversos hechos que ocurren en las empresas. Dado este amplio uso, el motivo por el cual aquí se estudia la cultura responde al afán de lograr la máxima precisión y exactitud en el análisis del concepto. Resulta confuso cómo se ha empleado el término hasta el momento, cuando se ha utilizado para denominar cualquier fenómeno en el que primen los aspectos subjetivos y reguladores del comportamiento.

La importancia de la cultura organizacional fue puesta en relevancia en el campo de la gestión y teoría organizacional a partir de la publicación del libro En búsqueda de la excelencia, de Peters y Waterman (1982). Su éxito radicó en el hecho de vincular el logro de los objetivos de la organización con la creación de una cultura organizacional fuerte.

Dentro de los estudios (Díez, 1999) que se han consultado, la relación cultura-estrategia se ha visto como una dependencia de la segunda para con la primera. La estrategia para lograr sus fines, depende de que la cultura lo favorezca o lo permita, si esta última no permite potenciar a la primera resulta imposible que se logren los resultados esperados. Una línea de pensamiento totalmente divergente con esta propuesta la brinda Díaz (2006), quien propone partir del estudio de la cultura organizacional como una forma de llevar adelante la estrategia desde su planificación.

Nuestro análisis, sin ser concluyente, nos lleva a comprender de una manera diferente esta relación. Se considera que estos son, en la práctica, categorías interpenetradas, multideterminadas, pero influenciadas definitivamente entre ellas. En la actualidad en el ámbito empresarial cubano no abundan los estudios de cultura en función de la dirección estratégica, así como tampoco se encuentran ejemplos que ilustren dentro de las estrategias el seguimiento a largo plazo de las variables e indicadores de la cultura organizacional, como forma de potenciar e intervenir en la misma.

Se propone, siendo las ideas propuestas con anterioridad por Díaz (2006), concebir esta relación como interdependiente. La única forma de desarrollar acertadamente la planeación estratégica de las organizaciones es, si y solo si, se tienen en cuenta la cultura y los distintos elementos que esta la compone. Este aspecto hace que las estrategias sean parti- 
culares, específicas y acorde a las reales características de la organización. Por otro lado, si dentro de la estrategia no se visiona los elementos de la cultura necesarios a transformar, la organización vivirá cerrada en su pasado, en sus logros anteriores, en sus creencias pasadas, al incluir estos elementos dentro de la estrategia se asegura que a la par de los resultados de índole económica, se desarroIlen los aspectos culturales y se incluyan por ejemplo elementos de cambio y otros necesarios a potenciar para alcanzar la visión. Mientras esto no se realice la distancia entre la misión y la visión será cada vez mayor ya que cuando transcurra el lapso de tiempo, veremos que la misión entonces se mantendrá, desde el punto de vista cultural, mientras que los elementos de la visión exigirán esfuerzos cada vez mayores en el entorno competitivo.

Con anterioridad se mencionó la posible existencia de barreras generadas por la cultura. Si se toma en cuenta en la estrategia la potenciación de la cultura, junto a otros aspectos esenciales en el desarrollo organizacional, a través de sus elementos integrantes (normas, valores, creencias, comportamientos, etc.) este efecto de barrera será minimizado, ya que para el desarrollo ulterior será necesario contar con esta cultura dinámica y flexible, enriquecida con nuevos o más fortalecidos elementos. La ventaja que traerá consigo la gestión de la cultura organizacional desde la estrategia, siendo consecuente con las ideas que se han esbozado, genera una empresa en constante crecimiento, con una estrategia ajustada a la medida del entorno y sus propias características, donde se potencia sus recursos, buscando siempre sal- tos cualitativos en torno a sus recursos intangibles y legitimando las etapas y logros de su constante desarrollo.

\section{Consideraciones finales}

Este análisis realizado permite enfocar el estudio de la cultura organizacional desde una perspectiva teórico-documental, haciendo un análisis factible y necesario en el contexto actual. La mayoría de las empresas de éxito apuestan a esta categoría como una de las vías de enfrentar las constantes transformaciones que se suceden día a día en el mundo contemporáneo. Desde el punto de vista científico, el debate se mantiene ya que aun no se logra la comprensión acabada de su naturaleza. Existe gran cantidad de conceptos y acercamientos a una realidad compleja, multideterminada y en constante evolución. Sin embargo en este estudio se pudo mostrar los elementos invariables dentro de este concepto, permitiendo realizar una valoración certera desde el punto de vista epistemológico.

La cultura organizacional engloba en sí a toda una serie de conceptos como creencias, valores, comportamientos, significados compartidos, entre otros. En este marco lógico se propone el concepto de rol como un elemento de vital importancia para el análisis de este tipo de cultura. Esta se manifiesta a través del rol en el individuo concreto y se hace palpable en las conductas también dentro de las funciones que el individuo tenga establecidos para sí en la empresa. Estas implicaciones operan en el plano teórico y metodológico del estudio de la cultura.

Otro aspecto trascendental a la hora de analizar la cultura en la práctica organi- 
zacional es la estrategia. La relación entre ellas, ha estado siendo comprendida de manera parcial. La interpenetración de ambas en el actuar de las organizaciones de hoy día son un nuevo reto para los directivos quienes tienen que comprender la necesidad de poner una en función de la otra, para potenciar y desarrollar en última instancia, a la organización.

\section{Referencias bibliográficas}

Aguirre Batzan, Angel (1996). La antropología aplicada a la empresa. Revista de Antropología Aplicada I/96, Instituto de Antropología de Barcelona, Barcelona, Pág. 42.

Bunge, Mario (2004). La ciencia. Su método y su filosofía. Escuela de Filosofía. Universidad ARCIS. Santiago de Chile. www.philosophia.cl. Fecha de Consulta: octubre del 2007.

Castro Ruz, Fidel (2007). El diálogo de las civilizaciones. Oficina de Publicaciones del Consejo de Estado. La Habana.

Chiavenato, Idalberto (1995). Introducción a la teoría general de la administración. Mc Graw Hill. Bogotá.

Deal, Terrence y Kennedy, Alan (1982). Corporate Culture: The Rites and rituals of corporate life. AddisonWesley. New York.

Díaz Llorca, Carlos (2006). Hacia una estrategia de valores en las organizaciones. Un enfoque paso a paso para los directivos y consultores. Serie Gerencial. Ediciones Balcón. La Habana.

Díez Gutiérrez, Enrique (1999). La estrategia del caracol. Un cambio cultural en una organización. OIKOS-TAU. Barcelona.

Fleury, María (1989). Cultura y poder en las organizaciones. Mc Graw Hill. Bogotá.
García, Salvador y Dolan, Simón (1997). Dirección por Valores. McGraw-Hill. Madrid.

Geertz, Clifford (1987). La interpretación de las culturas. Gediza. Barcelona.

Hatch, Mary (1993). The Dynamics of Organizational Culture. Academy of Management Review (October1993), pp. 657-693.

Katz, Daniel y Kahn, Robert (1986). Características que definen a las organizaciones sociales. En Psicología Social de las organizaciones. 5ta Ed. Trillas, México.

Mayo, Elton (1972). Problemas humanos de una civilización industrial. Nueva visión. Buenos Aires.

Nuñez, Jorge (2003). Problemas sociales de la ciencia y la tecnología. Félix Varela. La Habana.

Ohmae, Keniche (2005). The new global order of the world. Jorsey-Bass. San Francisco.

Ouchi, William (1982). Teoría Z: como pueden las empresas hacer frente al desafío japonés. Norma. Bogotá.

Peters, Tom y Waterman, Robert (1984). En búsqueda de la excelencia. Norma. Bogotá.

Real Academia Española de la Lengua (2001). Diccionario de la Real Academia de la Lengua Española. XXII Edición. Asociación de Academias de la Lengua Española. En: http://buscon.rae. es/drael/. Fecha de consulta: octubre del 2007.

Schein, Edgar (1986). Cultura organizacional y liderazgo, Jorsey-Bass. San Francisco.

Smircich, Linda (1983). Concepts of culture and organizacional analysis, Administrative Science Quarterly, No. 28. 